\title{
CANJE DE DEUDA POR NATURALEZA: LA NECESIDAD \\ DE UNA NUEVA AGENDA
}

\author{
Robert Devlin* \\ División de Desarrollo Económico \\ Documento de Trabajo No. $3 * *$ \\ Noviembre de 1991
}

* El autor es funcionario de la CEPAL. Las opiniones expresadas en este documento son de su exclusiva responsabilidad y pueden no coincidir con las de la Organización.

** Los trabajos incluidos en esta serie tienen por finalidad dar a conocer los resultados de las investigaciones en la CEPAL en forma preliminar a fin de estimular su análisis y sugerencias para su revisión. Esta publicación no es un documento oficial, por lo tanto no ha sido sometido a revisión editorial. Se puede solicitar directamente a la División de Desarrollo Económico de la CEPAL.

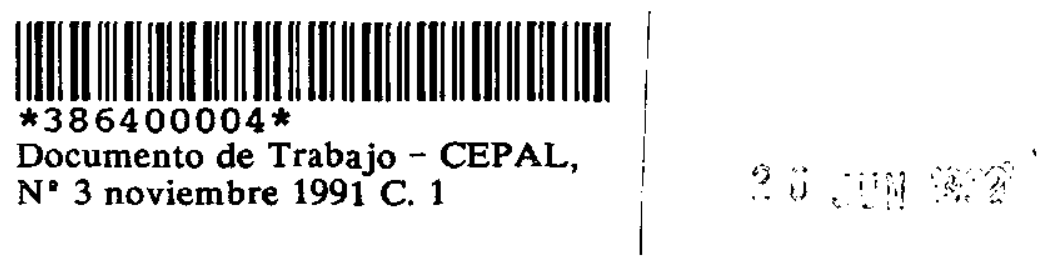



I N D I C E

Páginas

I. INTRODUCCION 1

II. LA EXPERIENCIA LATINOAMERICANA EN MATERIA DE CONVERSION DE DEUDA POR NATURALEZA

III. EL CANJE: UN PROBLEMA DEL RETORNO SOBRE EL USO ALTERNATIVO DE RECURSOS ESCASOS

IV. LA CONVERSION DE LA DEUDA BILATERAL

V. CONCLUSIONES

NOTAS

CUADROS 


\section{INTRODUCCIÓN*}

Durante la década de los setenta, América Latina acumuló una elevada deuda externa. Hasta 1982, las obligaciones externas de la región crecieron a un ritmo de más de $20 \%$ anual. Fue en ese año cuando finalmente explotó la comentada crisis externa, con una envergadura tal que afectó prácticamente a todos los pafses latinoamericanos.[1]

En 1982, la deuda externa de la región alcanzó a 330 mil millones de dólares, de los cuales más de las tres cuartas partes correspondieron a préstamos de la banca privada. Si bien esa deuda creció en los años posteriores, fue a un ritmo muy inferior comparado con la década precedente: se estima que la deuda externa latinoamericana alcanzó aproximadamente a 432 mil millones de dólares en 1990 y que el $55 \%$ de ese rotal corresponde a los bancos privados. El resto corresponde principalmente a obligaciones con organismos bilaterales y multilaterales.

A ráz de la crisis de pagos de la región, en los círculos financieros se generalizó la percepción de que por lo menos parte de la deuda pendiente era impagable. Así, casi desde el principio de la crisis, surgí un pequeño mercado informal secundario en el cual los bancos enfrentaron descuentos de 10 a $20 \%$ cuando intentaron vender o canjear sus pagarés. Como se sabe, los problemas de pago de la región se agudizaron a lo largo de la década, dando lugar a una acumulación de atrasos en el servicio de intereses de la deuda bancaria, la que alcanzó a alrededor de 21 mil millones de dólares a fines de 1990 (véase el cuadro 1). Con ello, los descuentos también se ampliaron; entre 1988 y 1990, se observó un precio promedio de la deuda latinoamericana en el mercado secundario de sólo 30-40 centavos por dólar (véase el cuadro 2). Por su parte, el volumen de transacciones en dicho mercado subió de $\&$ mil millones de dólares en 1985 a 65 mil millones de dólares en 1990 (véase el cuadro 3).

\footnotetext{
Agradezco los comentarios de Peter West, Patricio Mujica y Luis Alberto Santa Cruz sobre un primer borrador de este trabajo.
} 
La expansión del mercado secundario, junto con los crecientes descuentos registrados, incentivaron a los pafses para rescatar sus títulos de deuda a una fracción del valor nominal. Sin embargo, en vista de la escasez de reservas internacionales y las restricciones impuestas por los contratos de préstamo, hubo sólo oportunidades muy limitadas para efectuar una recompra directa de los pagarés que circulaban con descuento. No obstante, los países descubrieron que a través del patrocinio de programas de conversión con terceros que disponían de divisas, podrían indirectamente recomprar su deuda con activos denominados en moneda local. En 1985, Chile fue un pionero en el desarrollo del ya conocido mecanismo de conversión de la deuda en capital. En el segundo semestre de la década, otros países latinoamericanos establecieron sus propios esquemas de conversión de pagarés a capital accionario.[2] Este tipo de transacción claramente dominó los esquemas de reducción de la deuda en los años ochenta, alcanzando a 26 mil millones de dólares entre 1985 y 1990 . (Véase el cuadro 4).

La popularidad inicial de los programas de canje y el ofrecimiento de descuentos importantes propiciaron el surgimiento de otros esquemas. Uno de los que se ha puesto de actualidad es el denominado "canje de deuda por naturaleza". La idea se origino, aparentemente, en un artículo publicado por Thomas Lovejoy en el New York Times en el año 1984.[3] Por otra parte, y aunque el monto de este tipo de transacciones resulta todavía pequeño en comparación con el de las operaciones de conversión de deuda en capital accionario, el canje de deuda por naturaleza ha venido cobrando cada año mayor importancia debido al creciente interés mundial por la protección del medio ambiente.

El propósito del presente documento es mostrar algunos antecedentes sobre el canje de deuda por naturaleza y evaluar, en forma más bien general, el impacto de estas operaciones sobre el desarrollo de la región. 


\section{LA EXPERIENCIA LATINOAMERICANA EN MATERIA DE CONVERSIÓN DE DEUDA POR NATURALEZA}

Si bien los canjes de deuda resultan en la práctica bastante difíciles de organizar, conceptualmente son sencillos. Las etapas principales de esta transacción son las siguientes:

a) El pagaré de deuda externa de un país en desarrollo es adquirido con descuento en el mercado secundario, ya sea por el gobierno de un país industrializado; por una organización no gubernamental (ONG) dedicada a la conservación de la naturaleza o por algún organismo oficial.

b) El comprador extranjero entrega el pagaré al gobierno del pafs deudor a cambio de un instrumento financiero en moneda local (efectivo o bonos). Al efectuar el canje, el gobierno puede o no captar parte del descuento obtenido por el comprador en el mercado secundario.

c) El instrumento financiero en moneda local sirve para financiar un proyecto de conservación, previamente acordado entre el gobierno y la entidad extranjera. Lo común es que el proyecto sea administrado por grupos conservacionistas locales en colaboración con las autoridades gubernamentales del país.

Como se puede observar en el cuadro 5, a fines de 1990 se habían realizado once canjes de deuda por naturaleza en cinco países de América Latina. El monto nominal de la deuda rescatada llegó a casi cien millones de dólares. El costo de los pagarés para los compradores extranjeros fue de 17 centavos por dólar; es decir, captaron un descuento promedio de $83 \%$ en el mercado secundario. Por su parte, los gobiernos de la región rescataron la deuda a un precio equivalente a 59 centavos por dolar en moneda local, recibiendo de ese modo un descuento promedio de $41 \%$. Por otra parte, el mismo cuadro muestra que se realizaron otros canjes de deuda por naturaleza en países en desarrollo no latinoamericanos. 
Los proyectos de protección del medio ambiente financiados con canjes de deuda por naturaleza han tenido, por lo general, características bastante similares. Tienden a estar vinculados a la protección de terrenos conocidos por su riqueza biológica excepcional (flora y fauna) e incluso la presencia de especies amenazadas por extinción. Finalmente, dichos proyectos a menudo han incluido la creación o el reforzamiento de programas de educación, entrenamiento e investigación en materia de conservación ecológica.

Las zonas protegidas por los acuerdos siguen bajo la jurisdicción del país; sin embargo, suelen pasar a constituir zonas no desarrolladas o de desarrollo muy limitado.

Los estudios realizados sobre este tema tienden a afirmar que en los canjes de deuda por naturaleza, "todos los participantes se benefician"[4]. Por un lado, los bancos privados logran deshacerse de una parte problemática de su cartera a través de la venta del préstamo en el mercado secundario. Por otro, el organismo extranjero de conservación del medio ambiente logra multiplicar el impacto de su gasto, ya que, gracias al canje de los pagarés, recibe recursos en moneda nacional cuyo valor en dólares excede el monto inicialmente invertido en la operación de recompra en el mercado secundario. Con respecto al pars deudor, la operación de conversión cumple el doble propósito de reducir la deuda externa y de reforzar las políticas de protección del patrimonio nacional en materia de medio ambiente.

Cuando surgieron por primera vez las operaciones de conversión de deuda en capital, se solía justificarlas de manera similar. Sin embargo, con el tiempo comenzaron a estar sujetas a una evaluación más crítica que destacó algunos inconvenientes potenciales de estas operaciones para el país deudor.[5] En vista de que el canje de deuda por naturaleza es básicamente una variación sobre el mismo tema, se puede esperar que también merezca una interpretación más cautelosa. Los costos y beneficios que surgen de los canjes se hacen más evidentes al examinar analíticamente este tipo de transacción. 


\section{EL CANJE: UN PROBLEMA DEL RETORNO SOBRE EL USO ALTERNATIVO DE RECURSOS ESCASOS}

No cabe duda de que los canjes de deuda por naturaleza ofrecen importantes beneficios. Primero, en vista de los cuantiosos descuentos obtenidos en el mercado secundario, permiten que los organismos donantes aumenten el poder de compra de cada dólar en términos del valor nominal de la deuda que se puede rescatar. Por ejemplo, en 1987, en un canje para Ecuador, el organismo World Wildlife Fund (WWF) compró un millón de dólares de deuda a un costo de sólo 354 mil dólares (véase otra vez el cuadro 5). Además, esta inversión inicial movilizó el equivalente a un millón de dólares en moneda local para financiar la protección de la flora y fauna en más de dos millones de hectáreas de distintas reservas nacionales ubicadas a lo largo del pais[6]. Sin el efecto multiplicador del canje, es posible que el WWF hubiera tenido menos interés en asignar sus recursos al Ecuador.

Otro factor digno de destacar es que los canjes de deuda por naturaleza normalmente promueven el desarrollo de organismos locales de conservación. Son éstos, junto con el gobierno, los que colaboran con el donante extranjero para formular el proyecto de conservación. Suelen también participar en la administración y el financiamiento del proyecto. Ese fomento de la actividad de los grupos locales de conservación es sin duda uno de los aspectos más positivos del canje de deuda por naturaleza, por cuanto establece una base permanente para un "cabildeo verde" dentro del pás. Por último, el canje de deuda por naturaleza también logra una reducción de la deuda externa, incluyendo cualesquiera atrasos que se hayan acumulado sobre la misma.

Sin embargo, como contraparte de esos beneficios, existen también costos que se deberían tener en cuenta. En primer lugar, vale la pena recordar la existencia de literatura económica que demuestra que el rescate de pagarés en forma parcial y fragmentada no es necesariamente un buen negocio para un gobierno soberano.[7] El problema deriva del hecho de que cuando el precio de la deuda en el mercado secundario refleja razonablemente bien la probabilidad de que la deuda sea servida, el pars finalmente paga, en las operaciones de conversión, más de lo que vale el pagaré. En efecto, el precio al cual se transa la deuda es el del mercado; sin embargo, éste refleja el valor promedio de toda la suma de obligaciones (que incluye la deuda pagable y la no pagable). Asi, los 
pagares rescatados tendrían en el margen un valor inferior al precio que se paga en una operación de recompra en el mercado secundario[8]. Vista desde otro ángulo, la operación equivale a un subsidio a los bancos beneficiarios de la transacción marginal de recompra; por eso, algunos analistas se han referido al rescate parcial de la deuda por parte de los parses deudores en desarrollo como una conducta "manirrota".[9]

Existe un segundo subsidio derivado del hecho de que el pafs normalmente efectria el canje en condiciones en las cuales se entregan recursos en moneda nacional a un múltiplo del precio pagado por la deuda en el mercado secundario. Ya hemos observado que los países de América Latina canjearon el equivalente de 56 millones de dólares en moneda local contra pagarés de la deuda externa con un valor de 16 millones de dólares en el mercado secundario; ello representa un subsidio equivalente a 40 millones de dólares, o $42 \%$ del valor nominal de la deuda rescatada.

Es bien sabido, por otra parte, que las conversiones de la deuda provocan una expansión monetaria que puede tener efectos inflacionarios no deseados.[10] No obstante, existen maneras de minimizar el impacto de las conversiones sobre el nivel de precios internos; por ejemplo, muchos parses decidieron efectuar el canje a través de la entrega de bonos de mediano plazo. En efecto, el uso de bonos en las operaciones de canje distribuye la emisión monetaria a lo largo del tiempo; por lo demás, en algunos casos ha sido una manera indirecta de reducir el subsidio a los grupos conservacionistas involucrados en la conversión de la deuda.[11]

Por último, muchas veces no se ha tomado en consideración que un canje de pagarés por la naturaleza representa un pago adelantado de la deuda y, por ende, su impacto inicial es un aumento inmediato del gasto fiscal. Además, en la medida que el país deudor no esté sirviendo la deuda puntualmente --situación que todavía caracteriza a la mayoŕa de los parses latinoamericanos-- el efecto fiscal expansivo es más fuerte, ya que, desde el punto de vista del flujo de caja, no se produce un ahorro efectivo en el servicio de la deuda derivado de la cancelación de pagos de amortización e intereses. Esa presión sobre los egresos públicos, en una situación de ajuste estructural y de grave restricción fiscal, puede resultar altamente inconveniente.

Lo anterior sugiere que valdría la pena tomar en cuenta los costos, potencialmente importantes, que conllevan los canjes para el fisco del pars deudor si se considerara la posibilidad de 
concretar alguna de estas transacciones. A su vez, estos costos tienen que compararse con el carácter muy especial del beneficio principal esperado del canje de deuda por naturaleza: los proyectos de conservación financiados a través de este mecanismo suelen generar grandes externalidades internacionales, por lo que una parte importante de los retornos esperados son captados por la comunidad internacional. Es decir, sibien un proyecto de conservación de la flora y fauna nacionales --incluyendo la protección de especies al borde de la extinción-- tiene seguramente un retorno positivo para el país deudor, su impacto sobre la nación es normalmente de muy largo plazo.[12] De hecho, la tasa de descuento sobre ello --derivada de la función de bienestar de un país pobre en una crisis prolongada-- puede ser de tal magnitud que el beneficio para la comunidad internacional termine siendo bastante mayor que el recibido por la población del pars considerado. Por lo tanto, no es obvio que el aumento del gasto público y el otorgamiento de un subsidio para la conservación de la flora y fauna nacionales representen necesariamente la mejor asignación de los recursos escasos del estado cuando se atraviese, simultáneamente, por un período de grave crisis de desarrollo y una seria restricción fiscal. 


\section{LA CONVERSIÓN DE LA DEUDA BILATERAL}

Hasta ahora, los canjes de deuda por naturaleza se han realizado a través de la recompra y posterior conversión de títulos de la banca privada. No obstante, es probable que la conversión de la deuda oficial comience pronto a ser un vehículo para financiar proyectos de conservación del medio ambiente. Un síntoma de la nueva tendencia es el planteamiento sobre deuda externa contenido en la Iniciativa para las Américas del Gobierno de los Estados Unidos de América.[13]

En efecto, la Iniciativa contempla una reducción de la deuda de los países de la región con organismos oficiales de los Estados Unidos. Según la legislación presentada por el poder ejecutivo al Congreso, el Gobierno estadounidense estarla dispuesto a reducir la deuda concesional y permitir que los intereses correspondientes sean pagados en moneda local y depositados en fondos fiduciarios destinados a financiar proyectos nacionales de protección del medio ambiente. Con este propósito, el pais deudor tendría que negociar un acuerdo marco con el Gobierno de los Estados Unidos respecto a la administración y el uso de los fondos. Los proyectos conservacionistas tendrían que tener la aprobación de los dos gobiernos. Además, la Iniciativa pretende lograr una activa participación de los grupos locales de conservación en esas operaciones.

En cuanto a la deuda no concesional, el Gobierno estadounidense estaría dispuesto a vender, con descuento, parte de su cartera a compradores dispuestos a negociar luego canjes de la deuda por protección del medio ambiente. Otra vez, el uso de los fondos estaría sujeto a una consulta previa con las autoridades estadounidenses.

Mientras la deuda latinoamericana con el Gobierno de los Estados Unidos constituye una parte pequeña de la deuda total de la región (3\%), para algunos países en particular la participación es bastante significativa (veáse el cuadro 6). Asimismo, incluso algunos países con un monto relativamente reducido de obligaciones con los Estados Unidos podrían generar canjes de gran tamaño en relación con las transacciones del pasado.

Aparte de la polémica en torno a la fuerte condicionalidad contenida en la Iniciativa Bush [14], el programa de reducción de la deuda oficial para la protección del medio ambiente podría 
presentar algunos de los inconvenientes asociados con los canjes de títulos bancarios. Por una parte, la venta a grupos conservacionistas internacionales --con descuento de un pagaré de deuda no concesional-- para su posterior conversión aumentará inicialmente el gasto efectivo fiscal y podría involucrar el otorgamiento de un subsidio público a los conservacionistas extranjeros. Por otra parte, las operaciones de reducción de la deuda, tanto concesional como no concesional, inducen a una emisión monetaria potencialmente inconveniente. Por último, si el enfoque actual de los grupos conservacionistas internacionales -que han presionado mucho para la incorporación de la protección del medio ambiente en el plan de reducción de la deuda del Presidente Bush-- domina la Iniciativa, es de esperar que una gran parte de los beneficios de los proyectos financiados se transformen principalmente en externalidades para el beneficio de la comunidad internacional.

En cambio, algunos de los costos asociados con los canjes de deuda bancaria por naturaleza no se evidencian en el caso de una conversión de títulos de deuda con organismos oficiales estadounidenses. Por ejemplo, en la medida que se requiere que un país, para ser seleccionado, esté al día en el servicio de su deuda con el Gobierno de los Estados Unidos, la conversión de esa obligación generará una posterior reducción efectiva del gasto fiscal a través de menores pagos de amortización e intereses. Además, en la medida que no exista otra fórmula para reducir el valor actual de esas obligaciones externas sino mediante la participación en un programa estrechamente unido a la formulación de proyectos de protección del medio ambiente, el costo de oportunidad de la participación en este programa puede resultar relativamente bajo. En efecto, la única alternativa es enfrentar el costo total del servicio de esa deuda. En tales circunstancias, los beneficios generados para el pás deudor por el proyecto de conservación del medio ambiente, aunque bastante menores que los captados por la comunidad internacional, representan de todos modos un aporte positivo.[15]

Finalmente, en cuanto a la deuda oficial bilateral, vale la pena mencionar que los nuevos "Términos de Houston" del Club de París para la reestructuración de la deuda bilateral, disponibles para páses de ingreso medio-bajo, también contemplan una participación limitada en los programas de canje de tftulos por la naturaleza.[16] 


\section{CONCLUSIONES}

La conversión de la deuda en proyectos de protección del medio ambiente puede tener, además de beneficios, costos importantes para el fisco del país deudor. Estos costos se magnifican cuando el país está en medio de un duro proceso de ajuste estructural y escasean los recursos fiscales para financiar las transformaciones más básicas en materia de desarrollo y crecimiento económico. De esta manera, pese al gran entusiasmo que se ha observado recientemente en los cfrculos internacionales en torno a la protección del medio ambiente y de los canjes de deuda por naturaleza como mecanismo de su financiación, no es obvio que tales operaciones de conversión sean siempre favorables al país deudor. Por este motivo, cada transacción deberfa ser cuidadosamente evaluada en función de su impacto sobre la población nacional y a la luz de potenciales usos alternativos de los recursos escasos del estado. Después de un minucioso examen en términos estrictamente económicos y sociales del impacto sobre el país deudor de las distintas asignaciones posibles para los recursos del sector público, es posible que se concluya que muchos de los canjes más favorecidos por los grupos internacionales de conservación no ocupan un sitial alto en el rango de prioridades del programa nacional de desarrollo.

Sin embargo, se podrían superar algunos de los problemas más significativos asociados con los canjes si se lograra modificar la agenda internacional en esta materia. Cuando los organismos internacionales de conservación invierten en la protección del medio ambiente, buscan naturalmente proyectos que les den a ellos el mayor rendimiento posible. No es de sorprender, por lo tanto, que escojan habitualmente proyectos de gran perfil internacional, pues son aquéllos con los cuales identifican más claramente sus intereses y asocian una mayor rentabilidad. O sea, para dar un ejemplo más concreto, un programa de protección de los diez últimos cóndores de " $x$ " especie en el mundo --claramente un elemento del patrimonio internacional-- tendria, para el organismo extranjero, un rendimiento probablemente mayor que un proyecto de descontaminación del aire de la ciudad capital del pafs deudor. Es probable que la misma comparación, pero realizada desde el punto de vista del deudor, arrojaría los resultados exactamente contrarios.[17]

Esa asimetría de intereses entre el país deudor en desarrollo y el movimiento internacional de conservación no tiene por qué mantenerse en el largo plazo. En cambio en el corto y mediano 
plazos existen a menudo diferencias de preferencia en el tiempo, por lo que se deben buscar soluciones equitativas al respecto.

Con ese propósito, los países en desarrollo deben insistir en que los grupos internacionales de conservación amplíen su agenda e incluyan proyectos de protección del medio ambiente cuyos efectos se harán sentir en forma más amplia e inmediata sobre el bienestar de la población del pafs deudor. Ejemplos de este tipo de acción son la descontaminación del aire, de los ríos y del mar, cerca de los grandes centros urbanos o industriales de los paises en desarrollo, y la protección contra la erosión del suelo de zonas agrícolas. Este criterio cobra especial importancia cuando el proyecto de protección consume recursos fiscales escasos, como ocurre con los canjes de deuda por naturaleza. En este sentido, el Banco Interamericano de Desarrollo está desempeñando un papel de liderazgo a través de su propuesta de financiar un canje de deuda por naturaleza, en el cual el objetivo central es la reforestación de los alrededores de la Ciudad de México.[18]

En segundo lugar, cuando el proyecto de mayor interés para los organismos internacionales de conservación involucra la protección de lo que es en la práctica un cuasi-patrimonio internacional, existen posiblemente razones para insistir en que se realice mediante una donación directa, sin costo para el sector público. La donación se justifica por cuanto la mayor parte de los beneficios inmediatos del proyecto se transforman en externalidades para la comunidad internacional.

Por último, es diffcil evaluar los costos y los beneficios de los canjes de deuda por naturaleza para el país deudor. No cabe duda de que el creciente entusiasmo internacional para los proyectos de protección del medio ambiente es positivo y que, a fin de cuentas, sirve al interés común. Sin embargo, tal como en otros casos relacionados con bienes públicos, surgen problemas en torno a las externalidades, a la distribución de los beneficios y a quién debe pagar los costos. Aunque la protección del medio ambiente está convirtiéndose en el tema más en boga en los ćrculos internacionales, los gobiernos latinoamericanos no deberfan perder de vista el hecho de que los canjes de deuda por naturaleza sólo representan una dimensión adicional del dilema central de la presente crisis: ¿cómo asignar mejor los escasos recursos fiscales durante un período de ajustes severos y bajos niveles de actividad economica? 
$\underline{\text { Notas }}$

[1]

Para mayores antecedentes sobre la crisis, véase Robert Devlin, Debt and Crisis in Latin America: The Supply Side of the Story, Princeton, New Jersey, Princeton University Press, 1989

[2]

LDC Debt Reporter, octubre de 1990.

Véanse, Eugenio Lahera, "La conversión de la deuda externa vista desde América Latina", Revista de la CEPAL, No. 32, octubre de 1987 y Michael Mortimore, Debt Equity Conversion Programs: Guidelines for Debtors, Naciones Unidas, Centro de las Naciones Unidas sobre Empresas Transnacionales, Nueva York, febrero de 1989.

Véase Roque Sevilla Larrea y Alvaro Umaña Quesada, "¿Por qué canjear deuda por naturaleza?", Fundación Natura y Ministerio de Recursos Naturales de Costa Rica, San José y Quito, febrero de 1989.

[5]

Véanse Grupo de los Treinta, Financing for Developing Countries, Nueva York, 1987, y Ricardo Ffrench-Davis, "An interview with Ricardo Ffrench-Davis", en CTC Reporter, No. 23, Nueva York, segundo trimestre de 1987.

[6]

Véase Roque Sevilla Larrea, "El canje de la deuda por conservación en América Latina y el Caribe", Programa de las Naciones Unidas para el Medio Ambiente, Brasilia, marzo de 1989.

[7]

Véase, por ejemplo, Jeremy Bülow y Kenneth Rogoff, "The Buyback Boondoggle", Brookings Papers on Economic Activity, No. 2, 1988, pp. 675-704.

A manera de ejemplo, supóngase que existen dos escenarios posibles que afecten la capacidad de pagar la deuda. En el primero, que es favorable, el país pagaría el $100 \%$ de su deuda; sin embargo, la probabilidad de llevar a cabo esta situación sería sólo de un $25 \%$. En el segundo escenario,de carácter desfavorable, el país pagarfa sólo 
un $25 \%$ de su deuda y la probabilidad en esta última situación sería de $75 \%$. De esta manera, el precio de la deuda del país en el mercado secundario sería el promedio ponderado de los dos escenarios, es decir, 44 centavos. Cuando el país se encuentre en el "buen escenario", una recompra de un dólar de deuda por $\mathbf{4 4}$ centavos ahorraría un dólar en el servicio de la deuda. Sin embargo, en vista de que el pago total de la deuda ocurre con una probabilidad de sólo $25 \%$, el ahorro esperado de la reducción marginal de la deuda es sólo de 25 centavos. Es decir, el país paga 44 centavos por una deuda con un valor de 25 centavos.

[9] Bülow y Rogoff, op.cit.

[10] Grupo de los Treinta, op.cit.

[11] Se reduce el subsidio a través de una tasa de interés que está por debajo del ritmo de inflación. Otra manera de reducir el subsidio ha sido el uso del tipo de cambio oficial para realizar la conversión de la deuda a bonos. Véase, por ejemplo, Michael Occhiolini, "Debt-for-Nature Swaps", Banco Mundial, Washington, D.C., marzo de 1990.

[12] Una excepción serfa que existiera una importante industria de turismo ecológico.

[13] Véase, "Enterprise for the Americas' Initiative Act of 1990", Mensaje del Presidente de los Estados Unidos, Washington D.C., 18 de septiembre de 1990.

Véase Peter Hakim, "Latin American policy reconsidered", Journal of Commerce, 19 de octubre de 1990.

[15]

Al parecer, la reducción de la deuda concesional estarfa vinculada directamente al financiamiento de proyectos de protección del medio ambiente. En cambio, la reducción de la deuda no concesional se vincula a la opción de financiar proyectos de inversión en el sector privado o programas de conservación de la naturaleza. Así, en este último tipo de transacción, los países tendrían que evaluar cuidadosamente el 
costo de oportunidad de cada opción.

[16] En una reestructuración de la deuda del Club de París, los gobiernos acreedores pueden optar por un canje de la deuda por el capital accionario, la naturaleza, o el desarrollo. Todo la deuda concesional es elegible para dichas conversiones; en cuanto a las obligaciones no concesionales, se puede canjear hasta $10 \%$ o 10 millones de dólares, cualquiera que sea más grande.

[17] Como comento The Economist (16 de marzo de 1991, p.54), "...lamayoría de los brasileños son más proclives a eliminar la contaminación del aire de la ciudad que a detener la quema de árboles en el lejano Amazonas".

[18] Latin America Weekly Report, 4 de julio de 1991, p.7. 
CUNOPO 1

GERICA LATINA: ATRASOS DE IMTERESES STERE LA OELDA BAHCARIA

(HitLOMES DE DOLARES)

\begin{tabular}{|c|c|c|c|c|c|c|c|c|c|}
\hline PAISES & 1982 & 1983 & 1984 & 1985 & 1986 & 1987 & 1988 & 1989 & 1990 \\
\hline TOTAL & 1244 & 3453 & 2228 & 1222 & 1518 & 5878 & 5417 & 13557 & 20347 \\
\hline $\begin{array}{l}\text { EXPORIADSRES OE } \\
\text { PETROLEO }\end{array}$ & 51 & 145 & 114 & 743 & 1204 & 2042 & 2986 & 3944 & 4796 \\
\hline Bol IVIA & 51 & $n$ & 120 & 213 & 260 & 317 & 236 & 193 & 156 \\
\hline Colotgis & - & - & - & 64 & - & & & & \\
\hline ECUAODR & - & - & - & - & - & 368 & 804 & 1243 & 1544 \\
\hline Mexico & - & & & & & & & & \\
\hline PERU & - & 68 & 294 & 466 & 944 & 1357 & 1946 & 2508 & 3096 \\
\hline Vene zUELA & - & - & - & - & - & - & - & - & - \\
\hline $\begin{array}{l}\text { HO EXPORIADORES OE } \\
\text { PETAOLEO }\end{array}$ & 937 & 3308 & 1763 & 442 & 260 & 3667 & 1965 & 8702 & 15265 \\
\hline ARGENTIMA & 937 & 968 & 1743 & 442 & 257 & 228 & 1949 & 5216 & 6823 \\
\hline BhazIL & - & 2340 & - & - & - & 3430 & - & 3454 & 8376 \\
\hline CHTLE & - & - & - & - & & & & & \\
\hline PABAGUAY & - & - & - & - & 3 & 8 & 18 & 32 & 66 \\
\hline UAUGUAY & - & - & - & - & - & - & - & - & - \\
\hline AMG FICA CENTRAL & & & & & & & & & \\
\hline Y EL CARIBE & 256 & - & 71 & 37 & 54 & 169 & 466 & 911 & 886 \\
\hline COSTA RICA & 242 & - & 45 & 1 & 54 & 154 & 248 & 372 & . \\
\hline EL SALYACOA & $\cdots$ & ..' & $\cdots$ & $\cdots$ & '.' & ... & $\cdots$ & $\cdots$ & $\cdots$ \\
\hline GutaknLA & $\cdots$ & $\cdots$ & $\cdots$ & $\cdots$ & $\cdots$ & $\cdots$ & $\cdots$ & $\cdots$ & $\cdots$ \\
\hline HAIII & $\cdots$ & $\cdots$ & $\cdots$ & $\cdots$ & $\cdots$ & $\cdots$ & $\cdots$ & $\cdots$ & $\cdots$ \\
\hline Homouras & $\cdots$ & $\cdots$ & $\cdots$ & $\cdots$ & $\cdots$ & $\cdots$ & $\cdots$ & $\cdots$ & $\cdots$ \\
\hline Nicaragua & $\ldots$ & $\cdots$ & $\cdots$ & $\cdots$ & $\cdots$ & $\cdots$ & $\because$ & \#. & $\cdots$ \\
\hline Panamu & & - & & & - & 15 & 218 & 472 & 728 \\
\hline REPublich DOMimicamk & 14 & - & 26 & 38 & - & - & - & 67 & 158 \\
\hline
\end{tabular}

fuente: Imstifuto of fimazess Inteanacionales. 
Cuadro 2

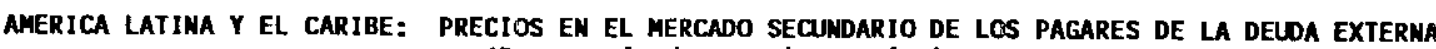
(Porcentaje de su valor nominal)

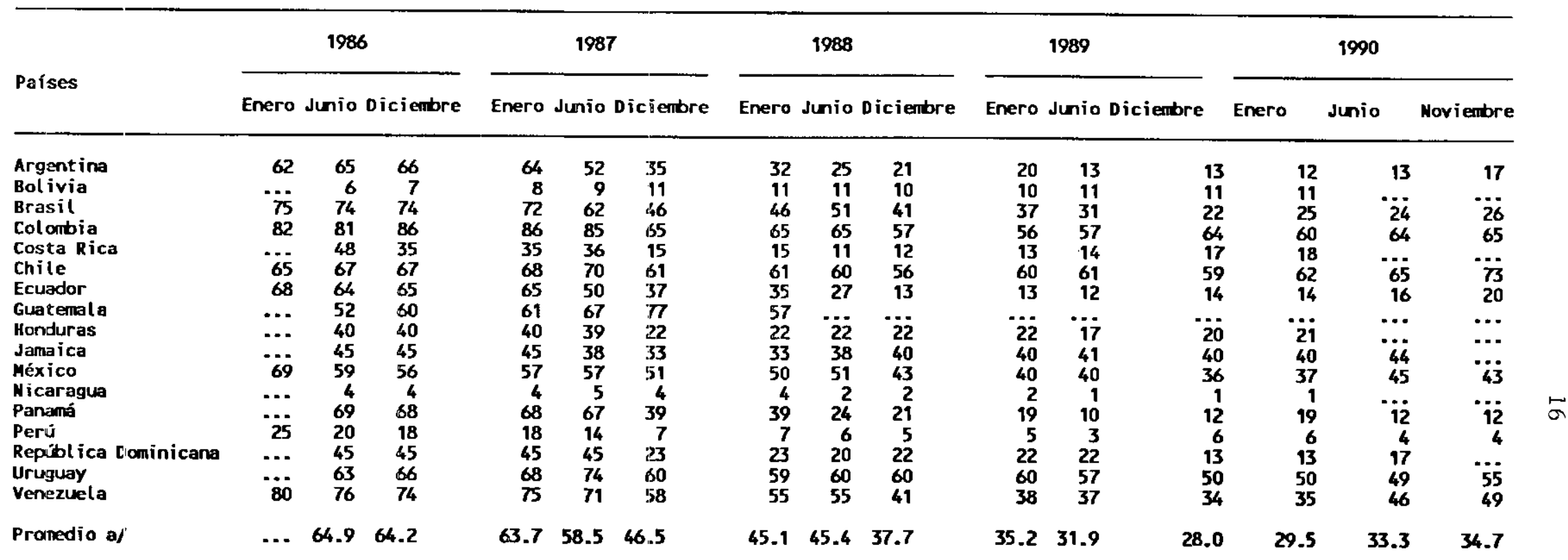

Fuente: Naciones Unidas, Departamento de Asuntos Económicos y Sociales Internacionales (DAESI), sobre la base de los precios de oferta ccmpilados por Salomon Brothers, High Yield Department.

a) Ponderaclo por el monto de la deuda bancaria. 
Cuadro 3

VOLUMEN DE TRANSACCIONES EN EL MERCADO SECUNDARIO

(en millones de dólares)

\begin{tabular}{llllllll}
\hline 1985 & 1986 & 1987 & 1988 & 1989 & 1990 & \\
\hline 4000 & 12000 & 50000 & 50000 & 60000 & 65000
\end{tabular}

Fuente: Banco Mundial, División de Deuda y Finanzas Internacionales, Quarterly Review, junio de 1991, P.12.

Cuadro 4

REDUCCION DE LA DEUDA

(en millones de dólares)

\begin{tabular}{lllllll}
\hline & 1985 & 1986 & 1987 & 1988 & 1989 & 1990 \\
\hline
\end{tabular}

\begin{tabular}{|c|c|c|c|c|c|c|}
\hline Total & 742 & 1601 & 7608 & $18 \quad 102$ & 12554 & 25283 \\
\hline $\begin{array}{l}\text { Conversión de la } \\
\text { deuda a capital }\end{array}$ & 497 & 822 & 3271 & 6794 & 4433 & 10589 \\
\hline $\begin{array}{l}\text { Conversión de la } \\
\text { deuda a moneda local }\end{array}$ & 156 & 438 & 796 & 1673 & 2428 & 2733 \\
\hline Recompra directa & 0 & 0 & 0 & 1862 & 1011 & 10696 \\
\hline Pago en moneda local & 0 & 63 & 87 & 3431 & 1569 & 512 \\
\hline $\begin{array}{l}\text { Reestructuración de } \\
\text { sector privado }\end{array}$ & 89 & 279 & 3454 & 4341 & 3113 & 500 \\
\hline
\end{tabular}

Fuente: Banco Mundial, División de Deuda y Finanzas Internacionales, Quarterly Review, junio de 1991, p. 12. 
Cuadro 5

AMERICA LATINA: CANJE DE DEUDA POR NATURALEZA

(Miles de dólares)

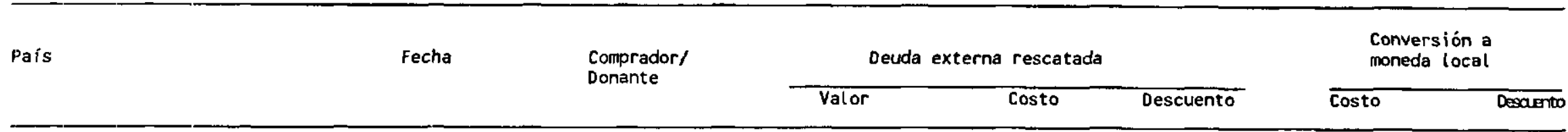

América Latina

$\begin{array}{ll}\text { Bolivia } & 1987 \\ \text { Ecuador } & 1987 \\ \text { Costa Rica } & 1988 \\ \text { Costa Rica } & 1988 \\ \text { Costa Rica } & 1989 \\ \text { Costa Rica } & 1989 \\ \text { Ecuador } & 1989 \\ \text { Repúbl ica Dominicana } & 1990 \\ \text { Costa Rica } & 1990 \\ \text { Mexico } & 1991 \\ \text { Costa Rica } & 1991\end{array}$

CI
WWF
FPN
Países Bajos
TNC
Suecia
WWF/TNC/MEG
PRCT/TNC
S/WWF/TNC
CI
RAA

$\begin{array}{rr}650 \\ 1 & 000 \\ 5 & 400 \\ 33 & 000 \\ 5 & 600 \\ 24 & 500 \\ 9 & 000 \\ 582 \\ 10 & 754 \\ 4 & 000 \\ 600\end{array}$

$\begin{array}{rr} & 100 \\ 354 \\ 918 \\ 5 \\ 5000 \\ 784 \\ 3500 \\ 1108 \\ 116 \\ 1954 \\ 1800 \\ 360\end{array}$

$85 \%$

$65 \%$
$83 \%$

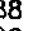

89

1991

RI

Promedio total

95086

15995

$85 \%$

$86 \%$
$86 \%$

$86 \%$

$88 \%$
$80 \%$

$82 \%$

$55 \%$
$40 \%$

Otros paises en desarrollo

$$
\begin{aligned}
& \text { Fil ipinas } \\
& \text { Madagascar } \\
& \text { Zambia } \\
& \text { Fil ipinas } \\
& \text { Madagascar } \\
& \text { Polonia }
\end{aligned}
$$

Filipinas

1989
1989
1989
1990
1990
1990
1991

1989

1989

1990

199

Promedio total

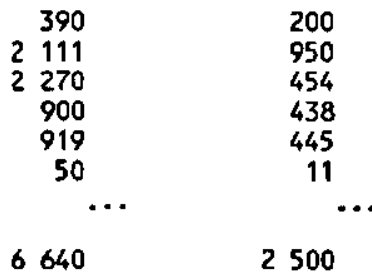

$83 \%$

$\begin{array}{rll}1 & 100 & 85 \% \\ 1000 & - \\ 4050 & 25 \% \\ 9900 & 70 \% \\ 1680 & 70 \% \\ 17100 & 30 \% \\ 9000 & - \\ 582 & - \\ 9603 & 11 \% \\ 2600 & 35 \% \\ 540 & 90 \%\end{array}$

$85 \%$

$5 \%$

$70 \%$

$30 \%$

$11 \%$

$90 \%$ Source: LDC Debt Report, 15 de octubre de 1990;Banco Mundial, Financial flows to Developing Countries, junio de 1991, y Ihe New York Times, 26 de

TNC : The Nature Conservancy

WWF : Horld Hildlife Fund

PRCT: Puerto Rico Conservation Trust

MBG : Missouri Botanical Garden

FNP : Fundación de Parques Nacionales de Costa Rica

C] : Conservation International

RA : Rain Forest Alliance 
Cuadro 6

DEUDA BILATERAL OFICIAL DE AMERICA LATINA Y

EL CARIBE CON LOS ESTADOS UNIDOS. 1990

(en millones de dólares)

\begin{tabular}{|c|c|c|c|}
\hline Países & $\begin{array}{l}\text { Deuda } \\
\text { total }\end{array}$ & $\begin{array}{l}\text { Deuda } \\
\text { bilateral }\end{array}$ & $\begin{array}{l}\text { Deuda } \\
\text { bilateral } \\
\text { oficial } \\
\text { (como \% de } \\
\text { total) }\end{array}$ \\
\hline
\end{tabular}

América

Latina $x$ el

Caribe

434800

12121

2.8

Países expor-

tadores de

petróleo

182376

4253

2.3

Bolivia

Col ombia

3774

16703

11241

Ecuador

98200

Mexico

17347

Trinidad y Tabago

2102

Venezuela

33009

536

1003

223

1625

732

114

20

14.2

6.0

2.0

1.7

4.2

5.4

Países no expor-

tadores de

petróleo

$\begin{array}{rl}216 & 750 \\ 65 & 000 \\ 122 & 200 \\ 18 & 602 \\ 1 & 802 \\ 1 & 763 \\ 7 & 383\end{array}$

3666

1.7

Argent ina

Brasil

Chile

Guyana

Paraguay

Uruguay

Centroamérica $y$

35674

el Caribe

Costa Rica

El Salvador

3700

2226

Guatemala

Hai 11

Honduras

Jamai ca

Nicaragua

Panama

Repüblica Dominicana
2602

861

3480

4152

8653

5700

4300
4252

12.0

524
2496

447

117

36

46

0.8

2.0

2.4

6.5

2.0

0.6

498

755

306

134

447

873

252

207

782
13.5

34.0

11.7

15.6

12.8

21.0

3.0

3.6

Fuente: CEPAL, sobre la base de cifras of iciales y SELA/JUNAC: "Evolución reciente de la dueda externa de América Lat ina y el Caribe", Lima, Perú, junio de 1991. 


